\title{
An Integrated Management Paradigm for Skull Base Chordoma Based on Clinical and Molecular Characteristics
}

\author{
Hussein M. Abdallah ${ }^{1}$ Zachary C. Gersey ${ }^{2} \quad$ Nallammai Muthiah ${ }^{1}$ Michael M. McDowell ${ }^{2}$ \\ Thomas Pearce $^{3}$ Tina Costacou ${ }^{4}$ Carl H. Snyderman ${ }^{5}$ Eric W. Wang ${ }^{5}$ Paul A. Gardner ${ }^{2}$ \\ Georgios A. Zenonos ${ }^{2}$
}

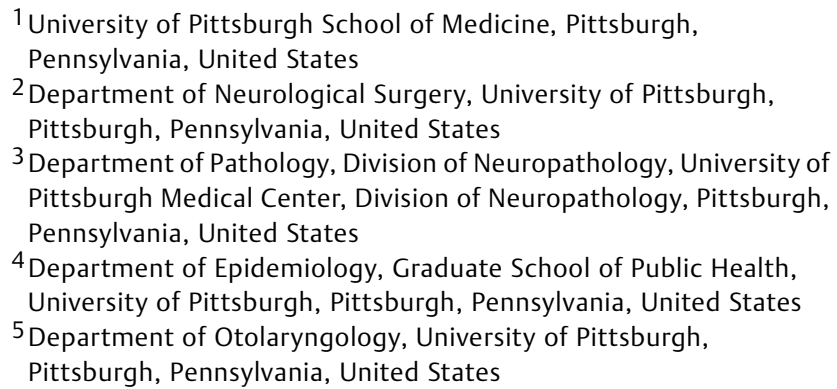

Address for correspondence Georgios A. Zenonos, MD, Department of Neurosurgery, Center for Cranial Base Surgery, University of Pittsburgh Medical Center, Pittsburgh PA 15213, United States (e-mail: zenonosg2@upmc.edu).

J Neurol Surg B Skull Base 2021;82:601-607.

\begin{abstract}
Keywords

- chordoma

- clival chordoma

- skull base

- genetics

- fluorescent in situ hybridization

- radiotherapy

Objective Previous work categorized skull base chordoma (SBC) into three genetic risk groups based on $1 \mathrm{p} 36$ and homozygous 9p21(p16) deletions, accounting for a wide variability in prognosis $(A=$ low-risk, $B=$ intermediate-risk, $C=$ high-risk). However, it remains unclear how these groups could guide management.

Study Design By integrating surgical outcome and adjuvant radiation (AdjXRT) information with genetic data on 152 tumors, we sought to develop an evidencebased management algorithm for SBC.

Results Gross total resections (GTRs) were associated with improved progression free survival (PFS) in all genetic groups. For Group C tumors, GTR and AdjXRT independently contributed to PFS (multivariate Cox proportional hazard ratio $[H R]=0.14, p=0.002$, and $\mathrm{HR}=0.40, p=0.047$, respectively). For Group B tumors, AdjXRT improved outcomes only when GTR was not feasible (log-rank $p=0.008)$, but not following GTR (log-rank $p=0.54)$. However, 24 of 25 Group A tumors underwent GTR, and AdjXRT for these did not confer any benefit $(\log -\operatorname{Rank} p=0.285)$. The high GTR rates in Group A could be explained by smaller tumor sizes (mean $=0.98 \mathrm{cc} / 4.08 \mathrm{cc} / 4.92 \mathrm{cc}$ for Group $\mathrm{A} / \mathrm{B} / \mathrm{C}$, respectively, $\mathrm{p}=0.031$ ) and lack of invasiveness. Group A tumors were also more frequently diagnosed in young people $(p=0.002)$ as asymptomatic lesions $(p=0.001)$, suggesting that they could be precursors to tumors in higher risk groups.

Conclusion Genotypic grouping by $1 \mathrm{p} 36$ and homozygous 9p21(p16) deletions can predict prognosis in SBC and guide management. GTR remains the cornerstone of SBC treatment and can be sufficient without AdjXRT in low and intermediate risk tumors. Low-risk tumors are associated with a less invasive phenotype, which makes them more amenable to GTR.
\end{abstract}

received

February 11, 2021

accepted

April 23, 2021

published online

June 22, 2021 (c) 2021. Thieme. All rights reserved.

Georg Thieme Verlag KG,

Rüdigerstraße 14,

70469 Stuttgart, Germany
DOI https://doi.org/

10.1055/s-0041-1730958. ISSN 2193-6331. 


\section{Introduction}

Skull base chordoma (SBC) is a rare, aggressive tumor of notochordal origin which occurs in the clivus and craniocervical junction, with an annual incidence of less than one per million in the United States. ${ }^{1-3}$ This rarity has been a major challenge in understanding their biology, which in turn has hindered the development of reliable classification schemes to account for their diverse clinical behavior and guide management. It is well established that chordomas lie on a diverse spectrum between indolent masses and rapidly lethal tumors. ${ }^{1-4}$ However, even in the most updated version of the World Health Organization manual, ${ }^{5}$ the classification relies mainly on histology, which does not fully account for the wide variability in prognosis. ${ }^{6,7}$

Due to the proximity of SCB lesions to cranial nerves and cerebral vasculature, these tumors are often not amenable to en bloc resection with oncologic margins. As they are typically resistant to standard chemotherapeutic agents, ${ }^{8,9}$ the mainstay of treatment has been maximal safe resection followed by high-dose adjuvant radiotherapy directed at microscopic and/or unresectable macroscopic remnants, despite the tumors being relatively radioresistant. ${ }^{9-11}$ While the morbidity of adjuvant radiation may be minimized in experienced centers, it is still associated with significant risks. ${ }^{12}$ In addition, it is currently unknown which chordomas truly benefit from adjuvant radiation, and if there are others where a gross total resection (GTR) is sufficient. Furthermore, there are no clear guidelines for tumors pre- dicted to have an aggressive clinical course for early enrollment in clinical trials with investigational pharmacotherapy protocols.

The molecular roots of these disparate survival patterns have been elusive. Pursuant to this need, a molecular prognostication panel based on the presence of two chromosomal deletions detected by fluorescence in situ hybridization (FISH), 1p36 and homozygous 9p21 (p16) deletions, was recently proposed. ${ }^{13}$ Based on the percentage of tumor cells with chromosomal deletions, the panel assigned patients into one of three risk groups: Group A (1p36: $<15 \%$ and 9p21: $<4 \%)$ representing the best prognosis, Group C (1p36: $>15 \%$ and $9 \mathrm{p} 21:>25 \%)$ representing the worst prognosis, and Group B (all other combinations of 1p36 and 9p21 percent deletions) representing an intermediate prognosis. This panel accounted for a wide range of cumulative hazard ratios for progression free survival (PFS) after surgery (1-56) and PFS after radiation (1-76), suggesting a strong link between these genotypic signatures and patient outcomes. ${ }^{13}$

While management recommendations were made in the aforementioned risk stratification schema, limited statistical power precluded the incorporation of surgical outcome or adjuvant radiation therapy data in the suggested guidelines, which were, therefore, not evidence based. In this current work, the larger number of cases and expanded follow-up allowed subclassification of cases according to the extent of resection and the administration of adjuvant radiotherapy with enough statistical power to provide evidence-based management recommendations.

Table 1 Cohort and tumor characteristics

\begin{tabular}{|c|c|c|c|c|}
\hline & Group A & Group B & Group C & $\begin{array}{l}p \text {-Value } \\
\text { (ANOVA or } \\
\text { Chi-square test) }\end{array}$ \\
\hline$n$ & 25 & 96 & 31 & \\
\hline Mean age at diagnosis (SD) & $36(14)$ & $49(18)$ & $41(17)$ & 0.002 \\
\hline Male sex & $14(56 \%)$ & $60(63 \%)$ & $22(71 \%)$ & 0.501 \\
\hline Mean tumor size, $\mathrm{cm}^{3}(\mathrm{SD})$ & $0.98(1.05)$ & $4.08(6.70)$ & $4.92(5.17)$ & 0.031 \\
\hline Recurrent on presentation & $12(48 \%)$ & $54(56 \%)$ & $24(77 \%)$ & 0.052 \\
\hline Asymptomatic at presentation (\%) & $11(44 \%)$ & $13(14 \%)$ & $3(10 \%)$ & 0.001 \\
\hline \multicolumn{5}{|l|}{ Involvement of } \\
\hline Upper clivus & $11(44 \%)$ & $58(60 \%)$ & $12(40 \%)$ & 0.084 \\
\hline Mid clivus & $22(88 \%)$ & $80(83 \%)$ & $16(53 \%)$ & 0.001 \\
\hline Lower clivus & $6(24 \%)$ & $42(44 \%)$ & $20(67 \%)$ & 0.006 \\
\hline Craniocervical junction & $5(20 \%)$ & $30(32 \%)$ & $20(67 \%)$ & $<0.001$ \\
\hline Two clival segments & $14(56 \%)$ & $74(77 \%)$ & $15(49 \%)$ & 0.005 \\
\hline Pan clival & $1(4 \%)$ & $16(17 \%)$ & $4(13 \%)$ & 0.249 \\
\hline \multicolumn{5}{|l|}{ Coronal plane extension } \\
\hline None & $18(75 \%)$ & $35(39 \%)$ & $7(23 \%)$ & $<0.001$ \\
\hline Unilateral & $5(21 \%)$ & $31(35 \%)$ & $15(50 \%)$ & 0.081 \\
\hline Bilateral & $1(4 \%)$ & $23(26 \%)$ & $8(27 \%)$ & 0.063 \\
\hline
\end{tabular}

Abbreviations: ANOVA, analysis of variance; SD, standard deviation. 


\section{Materials and Methods}

\section{Inclusion}

A retrospective review of clival chordoma cases at the University of Pittsburgh Medical Center revealed 152 cases containing complete clinical and FISH data for 1p36 and 9p21 deletions and were included in this study.

\section{Sample Acquisition}

This work was approved by an institutional review board (STUDY20040320) at the University of Pittsburgh. Surgical specimens from clival chordoma procedures performed between January 2007 and June 2020 were obtained and analyzed, and both primary and recurrent tumors were included (-Table $\mathbf{1}$ ). The pathological grading scheme and

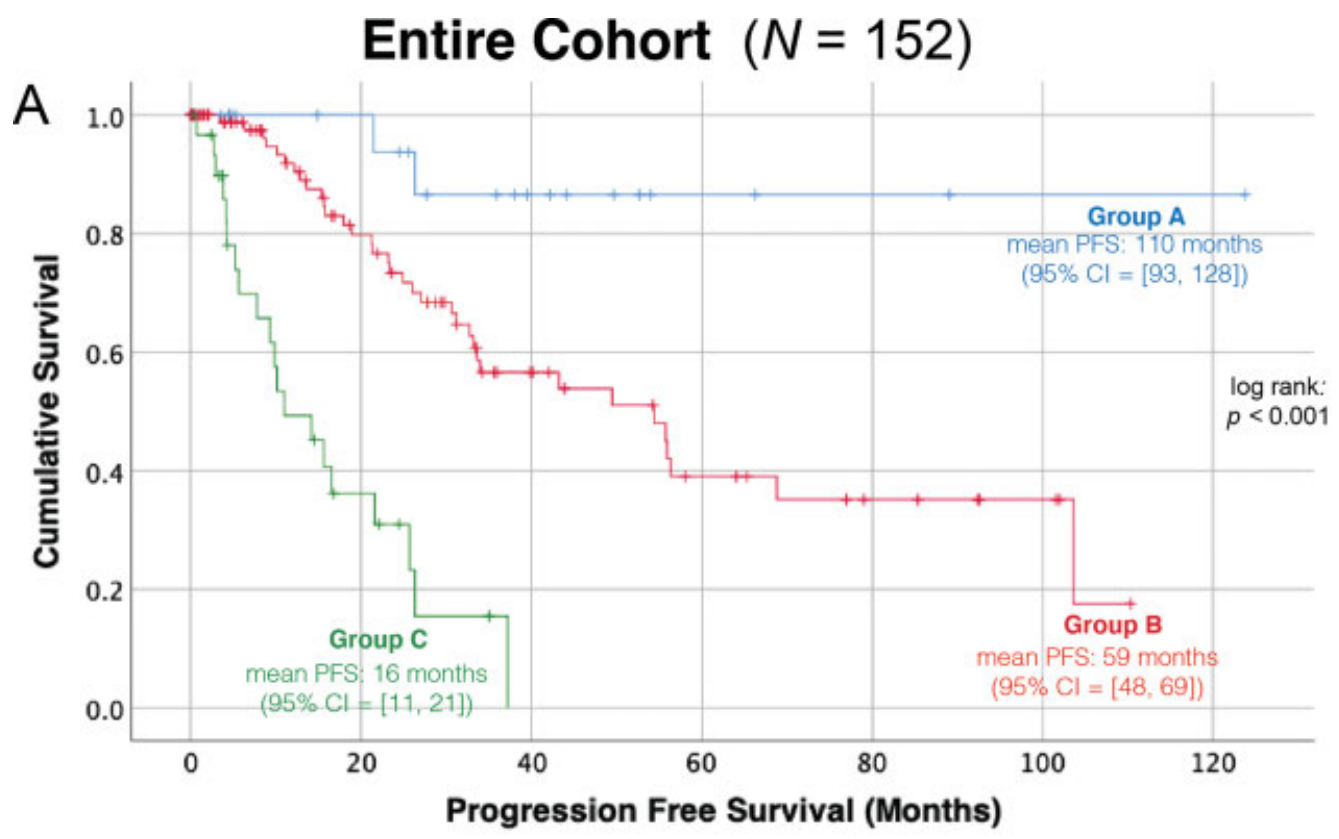

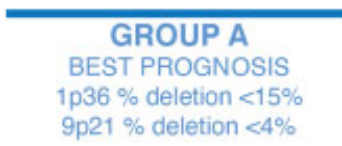

BEST PROGNOSIS

9 p21 \% deletion $<4 \%$

\section{GROUP B}

INTERMEDIATE PROGNOSIS

All other combinations of $1 \mathrm{p} 36$ and $9 p 21 \%$ deletion.

\section{GROUP C}

WORST PROGNOSIS

1 p36 $\%$ deletion $>15 \%$

9 p21 \% deletion $>25 \%$

\section{GTR Cases $(N=108)$}

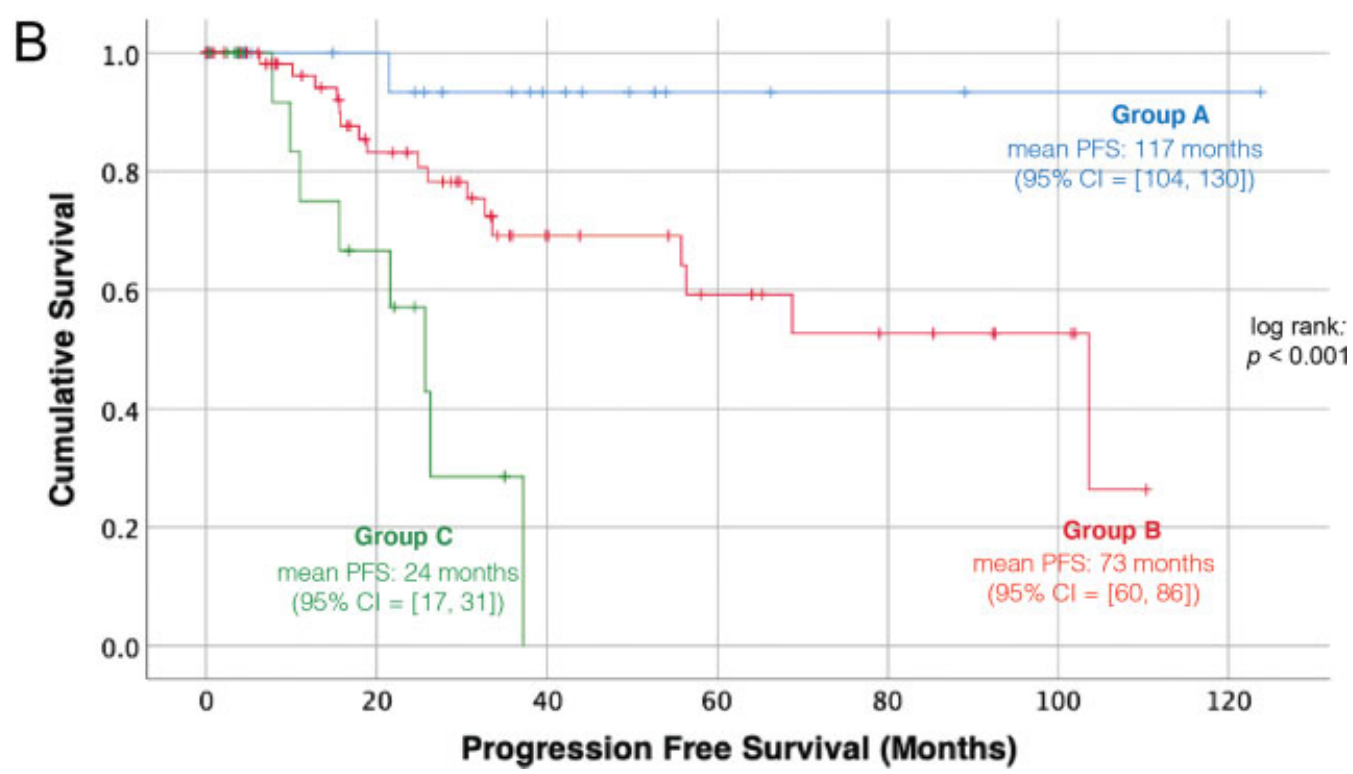

Fig. 1 PFS after surgery by genetic risk group. Kaplan-Meier PFS after surgery curves confirm that the observed differences in PFS between the three genetic risk groups when all cases are analyzed (A) persist regardless of the radicality of the surgical outcome (B). PFS, progression free survival. 


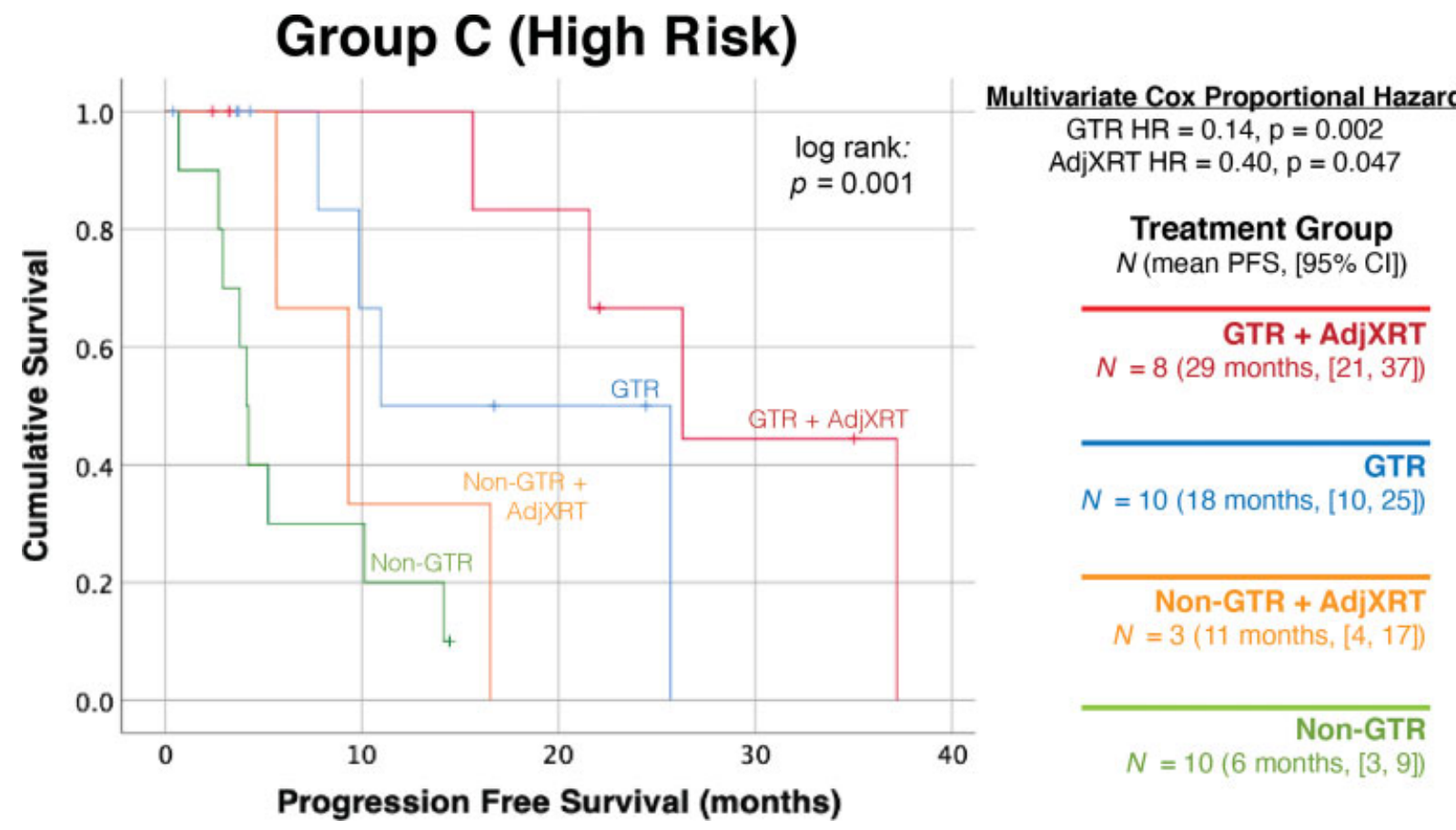

Fig. 2 Adjuvant radiation and GTR independently improve outcomes for high risk Group C tumors. A total of 31 cases were genetically classified as the highest risk tumors (1p36\% deletion: $>15 \%$, 9p21\% deletion: $>25 \%$ ). A multivariate Cox proportional hazard model including both GTR and adjuvant radiation demonstrated that both treatments independently improved outcomes, with GTRs having the largest effect. GTR, gross total resection.

FISH protocols utilized in this study were as described in previous work. ${ }^{13}$

\section{Statistical Analysis}

Survival analysis was performed for patients in each genetic risk group, with comparison arms that included the receipt of adjuvant radiation and achievement of GTR. Kaplan-Meier methods were used to estimate overall PFS after surgery determined with follow-up MRI imaging (always including volumetric contrasted sequences and $\mathrm{T} 2$ sequences), and logrank tests were used to calculate the equality of survival curves. Kaplan-Meier follow-up was defined as the time from the index surgery to either recurrence or the last imaging available with no evidence of recurrence. Both univariate and multivariable Cox regression analyses were performed to calculate hazard ratios for PFS after surgery. Bootstrapping with 1,000 samples was routinely part of each of these calculations. Where indicated, $t$-tests, ANOVA, and

\section{Group B (Intermediate Risk)}

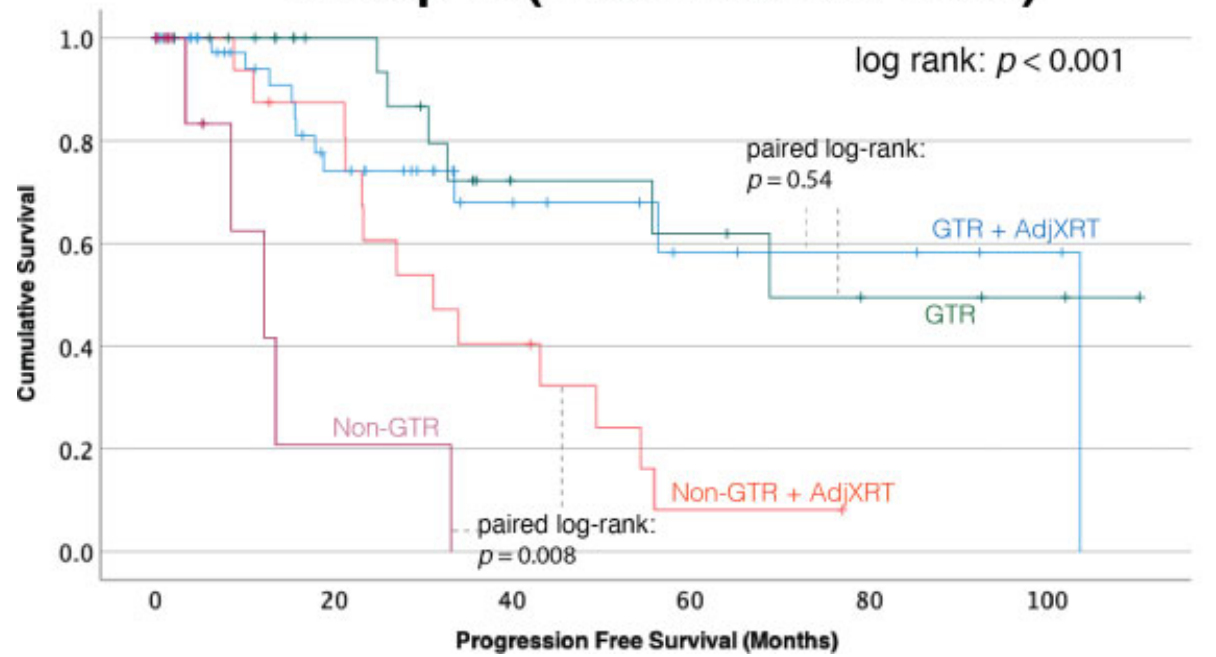

Treatment Group $N$ (mean PFS, [95\% Cl])

GTR + AdjXRT

$N=22(77$ months, $[57,97])$

GTR

$N=45$ (72 months, [54, 89])

Non-GTR + AdjXRT

$N=16(36$ months, $[26,46])$

Non-GTR

$N=13(15$ months, $[5,24])$

Fig. 3 Adjuvant radiation is only beneficial when GTR is not achieved in intermediate risk Group B tumors. A total of 96 cases were genetically classified as intermediate risk tumors based on chromosomal deletion percentages of 1p36 and 9p21, not meeting criteria for the other groups. Adjuvant radiation only provided additional benefit when GTR was not possible $(p=0.008)$, but did not provide any benefit if GTR was already achieved $(p=0.54)$. GTR, gross total resection. 


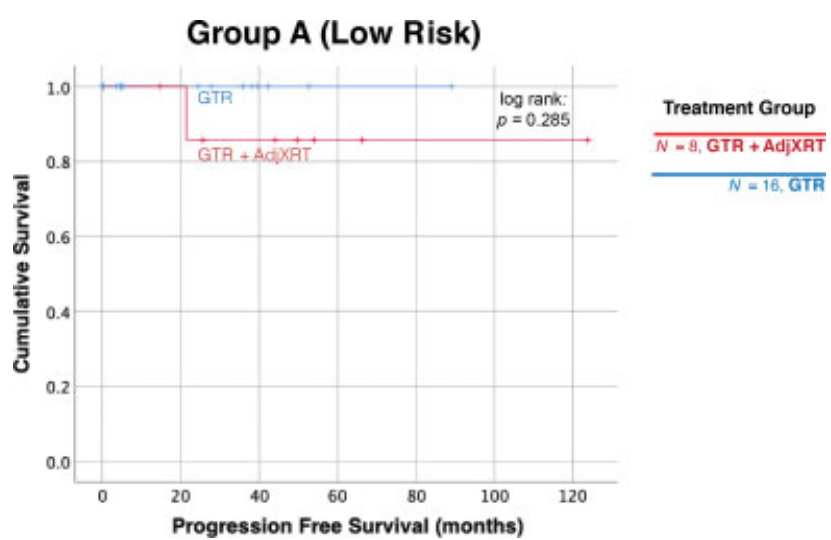

Fig. 4 Radiation therapy does not provide further benefit when GTR is achieved in Group A patients. There were 24 patients in Group A who received a GTR. Among these cases, adjuvant radiation did not have any benefit $(p=0.285)$. GTR, gross total resection.

Chi-square tests were used. All tests were two-sided, $p<0.05$ was considered statistically significant, and confidence intervals were set at the 95\% level. Statistical analyses were performed by using IBM SPSS (version 27, IBM Corp, Armonk, New York, United States).

\section{Results}

In the updated cohort of 152 cases, Kaplan-Meier survival estimation demonstrated that PFS after surgery was inversely proportional to the extent of chromosomal deletions captured by the genetic risk groups previously described ${ }^{13}$ (-Fig. 1A). Group A, B, and C patients were observed to have a mean PFS of 110 months (95\% confidence interval [CI]: 93-128), 59 months (95\% CI: 48-69), and 16 months (95\% CI: 11-21), respectively. Interestingly, even when only cases in which GTR was achieved were analyzed, the disparate survival patterns persisted: Group A, B, and C patients were observed to have a PFS of 117 months (95\% CI: 104-130), 73 months (95\% CI: 60-86), and 24 months (95\% CI: 17-31), respectively (-Fig. 1B). These trends lend further credence to the strength of tumor genotype in predicting patient prognosis.
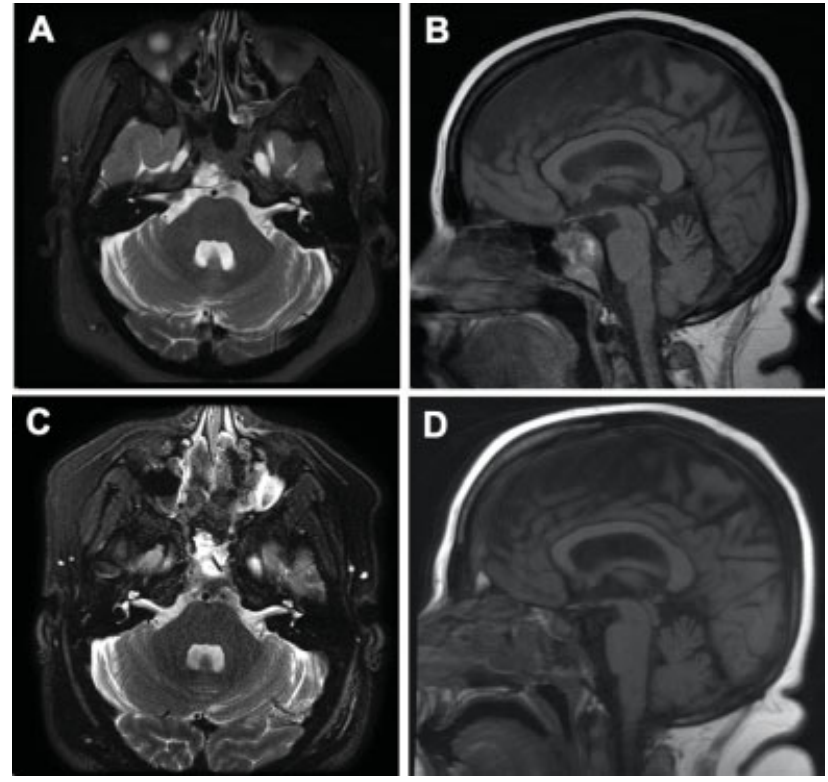

Fig. 5 Gross total resection of a representative Group A tumor. Preoperative axial (A) and sagittal (B) and postoperative axial (C) and sagittal (D) T2 magnetic resonance imaging showing gross-total resection.

The merits of adjuvant radiation and GTR were then analyzed separately within each genetic group. There were 31 cases in the highest risk genetic stratum (Group C), in which mean PFS varied from 6 months (95\% CI: 3-9) if neither GTR was achieved nor adjuvant radiotherapy administered to 29 months (95\% CI: 21-37), if a patient received both adjuvant radiation and GTR (-Fig. 2). Multivariate Cox proportional hazard ratio modeling of GTR and adjuvant radiation in this group showed that they both independently contributed to PFS, with the surgical outcome having the largest effect $(H R=0.14, p=0.002$ for $G T R$ and $H R=0.40$, $p=0.047$ for adjuvant radiation).

In the intermediate risk Group B, there were 96 cases with tumors containing chromosomal deletions that did not meet the criteria for either Group A or Group C. Mean PFS ranged between 15 months (95\% CI: 5-24) when neither GTR was achieved nor adjuvant radiotherapy administered to

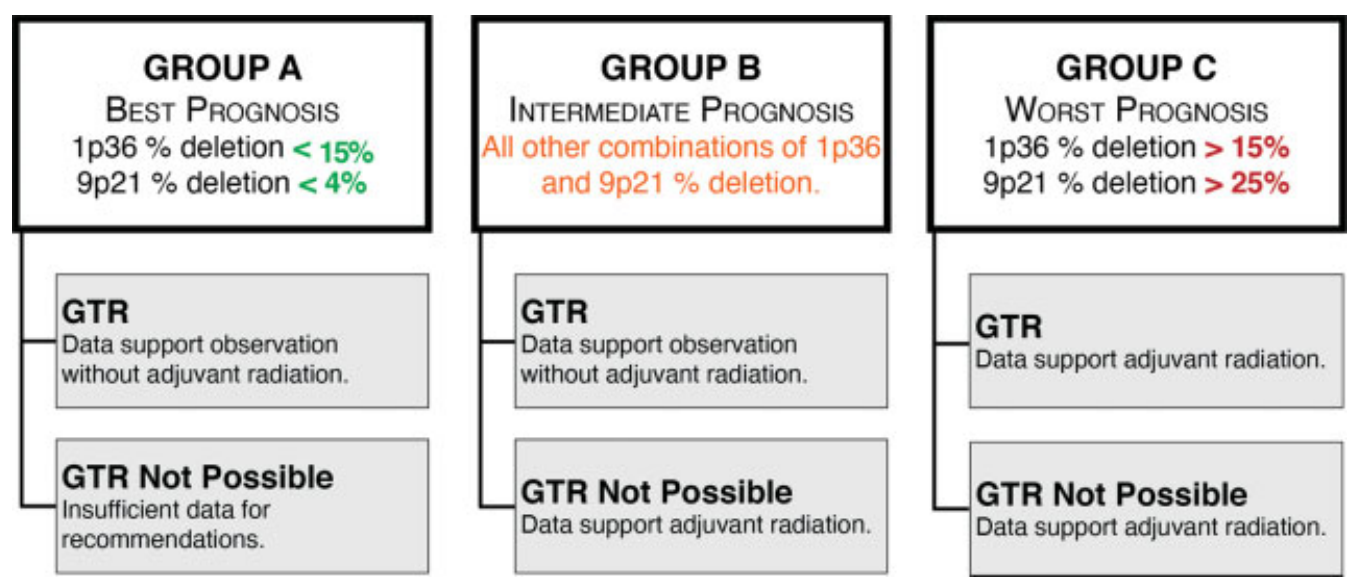

Fig. 6 An updated proposed management algorithm for skull base chordoma. 
77 months (95\% CI: 57-97) when both GTR was achieved and adjuvant radiotherapy was administered ( - Fig. $\mathbf{3}$ ). For these intermediate-risk tumors, adjuvant radiation only had merit when GTR was not possible (non-GTR with and without adjuvant radiation paired log-rank $p=0.008$ ), but did not provide any marginal benefit if GTR was already achieved (GTR with and without adjuvant radiation paired log-rank $p=0.54$ )

There were 25 cases in Group A, characterized by the relatively most favorable genetics-based risk. Virtually, all Group A cases received a GTR, with the exception of a single patient with tumor invasion into the brainstem. This patient did not receive radiotherapy, and the tumor progressed approximately 4 years after surgery, but the patient was subsequently lost to follow-up. This patient was excluded from the Kaplan-Meier survival calculations in Group A, which were performed to test the marginal impact of adjuvant radiotherapy when GTR was achieved. Among the GTR cases, adjuvant radiation did not confer any benefit $(p=0.285$, - Fig. 4). In addition, when compared with patients in Groups B and C, Group A patients were associated with a younger age at diagnosis, were more likely to be asymptomatic at presentation, and had tumors that were significantly smaller, had less frequent involvement of the lower clivus or cranio-cervical junction, and less frequent coronal plane extension ( - Table $\mathbf{1}$ ). - Fig. 5 depicts a representative case from this group in which GTR was achieved.

\section{Discussion}

\section{An Updated Management Algorithm for Skull Base Chordoma}

The current work reaffirms that SBC is a genotypically diverse type of tumor, and that much of the observed clinical variability in outcomes can be predicted by a relatively simple panel consisting of $1 \mathrm{p} 36$ and homozygous 9p21 (p16/CDKN2A) deletions detected by FISH. The prognostic significance of this panel persists even when eliminating surgical radicality as a factor. By substratifying the prognosis according to the surgical outcome and the use of adjuvant radiation, we were able to create an evidence-based management algorithm for SBC (-Fig. 6).

Patients undergoing GTRs fared better than patients in whom this was not feasible, and this was true within every genetic subgroup, and regardless of whether they received adjuvant radiation or not. As such, our data re-emphasize that radical resections remain the cornerstone of SBC treatment, and therefore, one or more approaches should be considered to achieve a GTR. For the tumors in Group C, associated with the worst prognosis, the data support the use of adjuvant radiotherapy for all patients, regardless of the best possible surgical outcome. In addition, given the overall dismal outcomes in this group, these patients should likely be counseled early about available clinical trials. This is especially true for patients in whom GTR is thought to be unlikely without significant morbidity, as such patients could potentially benefit from experimental protocols utilizing neoadjuvant treatments before further surgery is considered.
Adjuvant radiation is also indicated for intermediate risk tumors (Group B) in which a complete resection was not feasible, given that this was associated with a significantly better PFS after surgery compared with those tumors in which adjuvant radiation was withheld. By contrast, for patients in Group B in whom a GTR was achieved, adjuvant radiation did not confer a measurable improvement in progression-free survival. Therefore, it may be appropriate to withhold radiation and instead pursue rigorous surveillance. Given that intermediate prognosis tumors made up the majority of all cases, this recommendation may have a significant impact on how the largest number of chordomas are being treated, potentially sparing many patients of any radiation-associated morbidity.

Perhaps some of the most interesting results are related to the lowest risk genetic group. In addition to confirming their better overall prognosis, our data suggest that Group A tumors may be tied to a particular phenotype of tumor that is smaller, less invasive, asymptomatic when discovered, and discovered in younger patients. This constellation of findings begs the question of whether these tumors are benign precursors of those within the higher risk groups, analogous to lower grade gliomas undergoing malignant transformation later in life. In addition, our data suggest that this phenotype is associated with less invasive tumors that are more amenable to GTR. Indeed, all but one patient in Group A received a GTR in our cohort, and all of them had excellent prognosis regardless of adjuvant radiation. It is thus likely safe to suggest that for patients with complete resections in this group, adjuvant radiation can be withheld. It is also likely that such low-risk tumors significantly exaggerated the benefits of complete resections in many purely surgical chordoma series, ${ }^{14,15}$ as the phenotype which made these resections feasible was tied to a much more benign genotype. Given the paucity of cases without GTRs in this group, it is impossible to make clear management recommendations. The only patient without a GTR in this group experienced measurable progression on the gross residual tumor within the brainstem after approximately 4 years. The relatively longer time to show further growth is concordant with the more benign prognosis of Group A, and could suggest that close observation may not be completely unreasonable in patients in whom GTR is not feasible.

\section{Limitations}

The conclusions reached in this work should be taken in concert with several limitations. First, this is a single-institution study, and the results need to be validated using a larger multi-institutional set of samples. The determination of degree of resection can be subjective, and the surgical philosophy which led to these results includes radical resection of any and all grossly involved bone and dura. Less radical resections which might still be labeled GTR would likely not provide the same results. This should be considered when applying radiation recommendations. Second, as a retrospective work, this analysis may be influenced by selection bias. Finally, while this cohort is relatively large for a disease as rare as chordoma, the numbers are still 
limited, and more statistical power possible through multiinstitutional studies may reveal subtle differences which were undetectable in this study.

\section{Future Directions}

Validation of the current results in a multi-institutional series is critical for generalizability. Furthermore, combination of the current panel with other established markers could add further granularity to the proposed management paradigm. In addition, it could be useful to evaluate this panel in spinal chordoma.

\section{Conclusion}

Fluorescent in situ hybridization for $1 \mathrm{p} 36$ and homozygous 9p21 deletions is a relatively simple genetic panel capable of classifying SBC into three distinct prognostic groups that guide management. GTR should be the goal for all SBCs, and adjuvant radiation may be withheld in low or intermediate-risk tumors after undergoing GTRs. Conversely, adjuvant radiation was found to improve outcomes in patients with high-risk tumors regardless of the extent of resection, as well as in intermediaterisk tumors for which a GTR was not possible. Low risk tumors are more commonly incidentally found in younger patients and are relatively smaller and less invasive than other chordomas, making them more amenable to GTR.

Funding

H.M.A is supported by the NIH T35 training grant for student research in hematology and oncology.

Conflict of Interest

None declared.

\section{References}

1 McMaster ML, Goldstein AM, Bromley CM, Ishibe N, Parry DM. Chordoma: incidence and survival patterns in the United States, 1973-1995. Cancer Causes Control 2001;12(01):1-11
2 Chambers KJ, Lin DT, Meier J, Remenschneider A, Herr M, Gray ST. Incidence and survival patterns of cranial chordoma in the United States. Laryngoscope 2014;124(05):1097-1102

3 Smoll NR, Gautschi OP, Radovanovic I, Schaller K, Weber DC. Incidence and relative survival of chordomas: the standardized mortality ratio and the impact of chordomas on a population. Cancer 2013;119(11):2029-2037

4 Jones PS, Aghi MK, Muzikansky A, Shih HA, Barker FG II, Curry WT Jr. Outcomes and patterns of care in adult skull base chordomas from the Surveillance, Epidemiology, and End Results (SEER) database. J Clin Neurosci 2014;21(09):1490-1496

5 WHO. Soft Tissue and Bone Tumours, WHO Classification of Tumours, 5th Edition, Volume 3. Lyon, France: IARC Press; 2020

6 Bohman LE, Koch M, Bailey RL, Alonso-Basanta M, Lee JY. Skull base chordoma and chondrosarcoma: influence of clinical and demographic factors on prognosis: a SEER analysis. World Neurosurg 2014;82(05):806-814

7 Walcott BP, Nahed BV, Mohyeldin A, Coumans JV, Kahle KT, Ferreira MJ. Chordoma: current concepts, management, and future directions. Lancet Oncol 2012;13(02):e69-e76

8 Diaz RJ, Cusimano MD. The biological basis for modern treatment of chordoma. J Neurooncol 2011;104(02):411-422

9 Stacchiotti S, Sommer JChordoma Global Consensus Group. Building a global consensus approach to chordoma: a position paper from the medical and patient community. Lancet Oncol 2015;16 (02):e71-e83

10 Yaniv D, Soudry E, Strenov Y, Cohen MA, Mizrachi A. Skull base chordomas review of current treatment paradigms. World J Otorhinolaryngol Head Neck Surg 2020;6(02):125-131

11 Koutourousiou M, Gardner PA, Tormenti MJ, et al. Endoscopic endonasal approach for resection of cranial base chordomas: outcomes and learning curve. Neurosurgery 2012;71(03):614-624 , discussion 624-625

12 Alahmari M, Temel Y. Skull base chordoma treated with proton therapy: a systematic review. Surg Neurol Int 2019;10:96

13 Zenonos GA, Fernandez-Miranda JC, Mukherjee D, et al. Prospective validation of a molecular prognostication panel for clival chordoma. J Neurosurg 2018:1-10

14 Wang EW, Zanation AM, Gardner PA, et al. ICAR: endoscopic skullbase surgery. Int Forum Allergy Rhinol 2019;9(S3):S145-S365

15 Zenonos GAAK, Koutourousiou M, Zwagerman NT, Panczykowski D, Wang EW. Endoscopic endonasal approach for clival chordomas: 12 years of experience from a large skull base referral center. J Neurol Surg B Skull Base 2016;77:A007 